Ann. Zootech., I966, 15 (4), 303-3I2.

\author{
I.N.R.A. \\ BIBLIOTHEQUE UÓ 35906 \\ Domaine de CROUELLE \\ 63039 \\ Clermont-FD CeDeX 2
}

\title{
LES PARTICULES VÉGÉTALES DES FÈCES DE MOUTON
}

\author{
Élisabeth GRENE'T \\ Station de Recherches sur l'Élevage des Ruminants, \\ Centre national de Recherches zootechniques, 78 -Jouy-en-Josas
}

\section{SOMMAIRE}

Nous avons étudié la taille des particules fécales, la nature des particules végétales et la proportion de membranes lignifiées qu'elles renferment, dans 22 échantillons de fèces de mouton, en relation avec la composition chimique et la digestibilité du fourrage ingéré.

La taille moyenne des particules fécales est relativement faible : elle varie de o, 108 à $0,206 \mathrm{~mm}$. Elle augmente lorsque la digestibilité de la matière organique et de la cellulose brute des fourrages diminue. Flle varie dans le même sens que la teneur en cellulose brute et en matière organique indigestible des fourrages, et que la teneur en cellulose brute des fèces.

Les grosses particules, dont nous avons spécialement étudié la nature et la lignification, sont composées de fragments de tissu lignifié (vaisseaux, silérenchyme) ainsi que de lambeaux d'épiderme et de parenchyme. La présence de fragments d'épiderme est variable; elle dépend des espèces et des stades étudiés, mais nous en avons trouvé dans tous les échantillons de fèces d'automne.

La lignification des particules augmente au cours du rer cycle de croissance; elle varie en sens inverse de la digestibilité et dans le même sens que la teneur en cellulose brute du fourrage et des fèces.

\section{INTRODUCTION}

Les fèces de mouton sont composées de résidus végétaux non digérés et de déchets d'origine endogène tels que des sucs digestifs, des cellules du tube digestif et des produits d'origine microbienne. Leur composition chimique est liée à la digestibilité de la matière organique du fourrage ingéré (JARRIGE, I965).

Mais très peu d'études ont été faites jusqu'à présent sur la composition microscopique des fèces. L'épiderme de la feuille de graminée étant caractéristique d'une espèce donnée, il est possible par l'examen des fèces d'un animal d'identifier les plantes ingérées (CROKER, I959). HERCus (I960) en Nouvelle-Zélande, STORR (I96I) et Adams, O'Regax et Dunaway (1962) en Australie, Martin (I962) en Écosse, ont estimé par cette méthode la proportion de différentes espèces ingérées. 
Drapala, Raymond et Crampton (I947) et Steppler (I95I) au Canada, et REGAL (I960) en Tchécoslovaquie, à l'aide d'examens microscopiques, ont évalué la proportion de membranes lignifiées dans les plantes fourragères, et observé la nature des tissus retrouvés dans les fèces. D'autre part, afin de compléter les analyses chimiques, HELISTRÖM (I955-I958-1965) en Suède, a effectué un fractionnement mécanique des fèces à l'aide d'ultra-sons. Ces auteurs ont relié leurs résultats à la digestibilité du fourrage.

Nous avons étudié la taille des particules fécales de mouton, la nature des particules végétales et la proportion des membranes lignifiées qu'elles renferment. Nous avons mis en parallèle nos résultats avec la composition chimique et la digestibilité du fourrage ingéré.

\section{MATÉRIEI, E'T MÉTHODES}

\section{Choix et préparation des échantillons}

Une étude de la valeur alimentaire des fourrages des prairies temporaires est effectuée à la Station par C. Demaroquilly et nous avons profité des mesures de digestibilité qu'il poursuit pour préparer 22 échantillons de fèces de mouton. Ils correspondent à des fourrages de printemps coupés à différents stades de végétation du er $^{\mathrm{er}}$ cycle, et à des fourrages d'automne. Ces fourrages représentent les principales espèces de légumineuses et de graminées fourragères : luzerne, trèfle violet, fétuque des prés, ray-grass d'Italie et ray-grass anglais, fléole, dactyle. Le tableau I en donne les caractéristiques botaniques et morphologiques, ainsi que les coefficients de digestibilité de la matière organique et de la cellulose brute Weende (C. Demarquilly, communication personnelle).

Les fourrages ont été coupés chaque matin et distribués en 3 repas à des lots de 4 moutons de race Texel, âgés de 2 ans, maintenus en cage à métabolisme. Les moutons étaient alimentés ad libitum, la quantité distribuée étant ajustée chaque jour de façon que le pourcentage de refus soit de l'ordre de $10 \mathrm{p}$. 100. La période de mesure durait une semaine pour chaque échantillon.

Nous avons prélevé nos échantillons les deux derniers jours des périodes de mesure sur le mélange pondéré des fèces des 4 moutons. Ces échantillons ont été séchés à l'étuve à $70^{\circ} \mathrm{C}$ à ventilation forcée et n'ont pas été broyés.

Le tableau $\mathrm{I}$ indique la composition chimique des fèces : teneurs en cendres, matières azotées et cellulose brute Weende $p$. Ioo de la matière sèche; la quantité de matière sèche excrétée par jour et par kilogramme de poids métabolique est donnée dans le tableau 2.

\section{Séparation des particules fécales selon leur taille}

Trois prises de $2 \mathrm{~g}$ de fèces sont déshydratées à l'étuve à $80^{\circ} \mathrm{C}$ puis sont pesées et mises à tremper dans un cristallisoir avec $60 \mathrm{ml}$ environ d'eau (additionnée de ro p. roo de teepol) pendant au moins 24 heures, à la température ambiante. Elles sont transvasées dans un mortier où elles sont écrasées légèrement, puis tamisées en milieu liquide à l'aide d'une "tamiseuse " électromagnétique Fritsch, sur 3 tamis à maille d'ouverture respective : $0,400,0,160$ et $0,100 \mathrm{~mm}$. Chaque tamisage est effectué avec une amplitude de vibration voisine de $3 \mathrm{~mm}$ et à une fréquence de 3 ooo périodes par minute.

Entre chaque tamisage les résidus sont étalés régulièrement sur le tamis de $0,400 \mathrm{~mm}$ d'ouverture à l'aide d'une spatule, afin d'obtenir une bonne répartition des particules sur les mailles.

Pour la première série de ıo échantillons, le tamisage a été effectué pendant 25 minutes, divisé en périodes de 5 minutes. Le tamis supérieur était arrosé entre chaque période avec l'eau d'une pissette. La quantité d'eau employée était de $\mathrm{I}, 5$ litre en tout. Par la suite la " tamiseuse " fut équipée d'un couvercle avec tête à douche, et d'un fond de tamis avec une évacuation. Dès lors, le tamisage fut effectué pendant une durée de 20 minutes, divisée en périodes de 5 minutes. L'arrivée d'eau était ouverte à trois reprises de façon à arroser les tamis avec 1,5 litre de liquide à chaque fois.

Dans les deux cas l'eau d'arrosage est recueillie dans des béchers de 2 litres. La récupération des particules fécales sur les tamis s'effectue à l'aide d'un jet de pissette remplie d'eau, dans un bécher de un litre, puis le liquide contenant les particules en suspension est filtré sur un buchner garni d'un rond de papier filtre sans cendres, préalablement taré. Le résidu est séché à l'acétone, déshydraté à l'étuve à $80^{\circ} \mathrm{C}$ pendant 48 heures et pesé. 


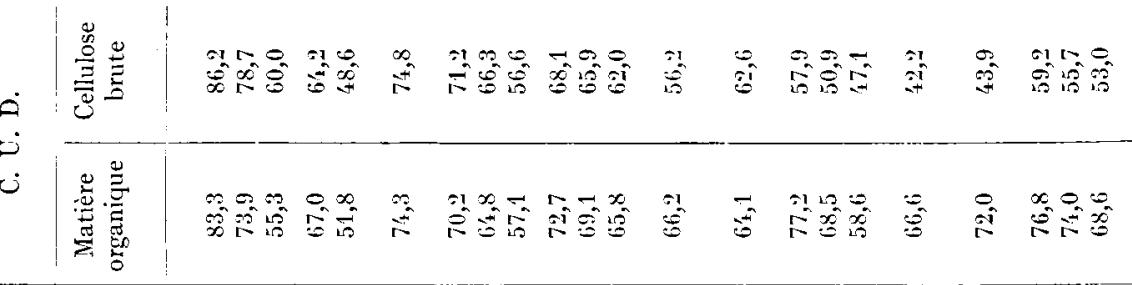

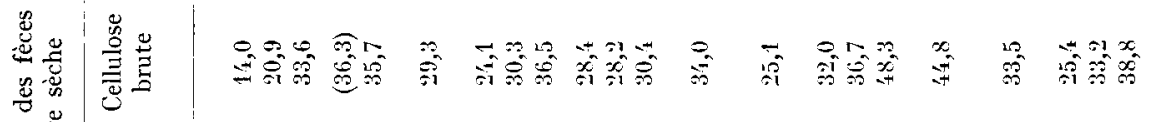

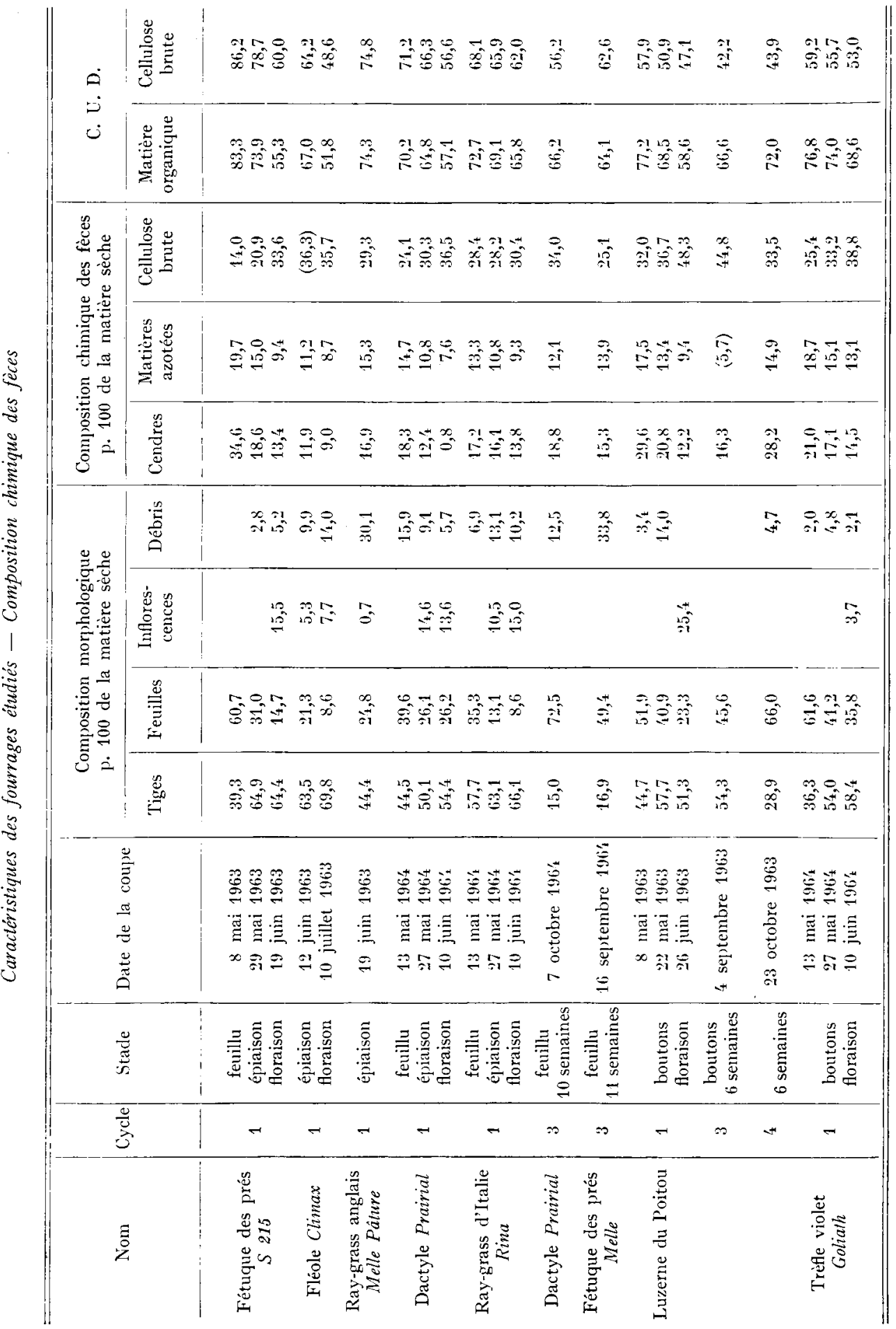


L'eau d'écoulement contenant les particules les plus fines en suspension est filtrée sur deux filtres plissés tarés, superposés. Le résidu est séché et pesé comme précédemment.

\section{Examen microscopique des particules fécales}

Les particules fécales de taille supérieure à $0,400 \mathrm{~mm}$ sont soumises à une double coloration, à la safranine qui colore les tissus lignifiés en rouge, et au vert rapide qui colore les tissus cellulosiques en vert.

Pour cela elles sont mises en suspension dans de l'eau additionnée de quelques gouttes de teepol et l'ensemble est placé sur un agitateur magnétique. Quelques gouttes de liquide sont prélevées et placées dans un petit "panier ", poche de tissu de nylon à mailles fines montée sur une armature de fil de laiton. La coloration à la safranine et au vert rapide est alors effectuée selon la technique de D. A. Johansen (1940), avec les modifications suivantes : le premier bain d'alcool à 35 p. roo est remplacé par de l'alcool à $40^{\circ}$, et la safranine est en solution à I p. Ioo dans l'alcool à $95^{\circ}$; cette solution est diluée au moment de l'emploi avec un égal volume d'eau distillée.

Un petit nombre de particules prises à l'aide d'un pinceau fin, est monté au baume du Canada, entre lame et lamelle : io préparations sont examinées pour chaque échantillon. Sur chaque préparation le nombre de particules est compté, et une note est attribuée à chaque particule, variant de o à Io suivant la proportion de tissus lignifiés par rapport aux tissus cellulosiques différenciés par la double coloration. Chaque préparation faisant l'objet de deux comptages, nous avons donc calculé la moyenne de 20 notes pour chaque échantillon.

\section{RÉSULTATS}

Afin de simplifier l'exposé des résultats nous adopterons la convention suivante : les fèces de moutons alimentés avec des légumineuses par exemple, seront appelées "fèces de légumineuses". Il en sera de même pour chaque catégorie de fèces.

\section{Répartition des particules fécales selon leur taille. Taille moyenne}

Le tableau 2 donne les pourcentages de la matière sèche fécale recueillie sur les différents tamis de 0,400 , o,I 60 et 0 , I0o $\mathrm{mm}$ d'ouverture et dans le fond de tamis.

La matière sèche totale recouvrée est inférieure de 5 à $\mathrm{I} 8 \mathrm{p}$. Ioo à la matière sèche tamisée. La différence correspond aux substances solubles dans l'eau, aux pigments et matières grasses dissous par l'acétone sur le filtre, et aux particules extrêmement fines qui ont pu traverser les filtres.

La répartition de la matière sèche varie au cours de la croissance du fourrage. D'une manière générale la proportion retenue par les tamis de 0,400 et $0,160 \mathrm{~mm}$ d'ouverture augmente au cours du I er cycle de croissance, tandis que celle qui est retenue par le tamis de 0, IOo varie peu. Le pourcentage de la matière sèche fécale qui est retenu par les trois tamis croît régulièrement au cours du I er cycle, au détriment de celui qui est recueilli dans le fond de tamis; de moins de $50 \mathrm{p}$. roo au début du cycle (4I p. IOo) pour la fétuque, il passe à $67 \mathrm{p}$. IoO (et même $72 \mathrm{p}$. Ioo pour la fléole) à la floraison. A stade égal il diffère selon la précocité des plantes.

Nous avons calculé la taille moyenne pondérée des particules fécales en supposant que toutes les particules retenues sur un tamis ont la taille de la maille de ce tamis, et que celles qui sont recueillies dans le fond de tamis ont une taille moyenne de $0,050 \mathrm{~mm}$. 


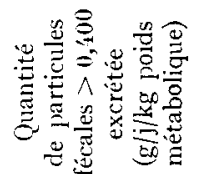

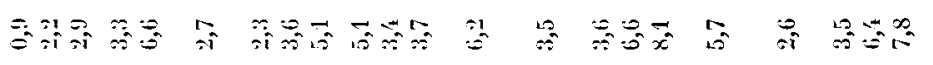

苛

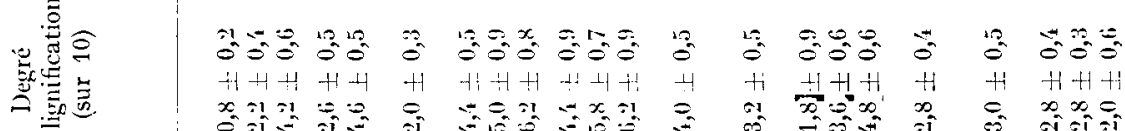
$\approx$

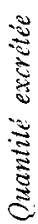

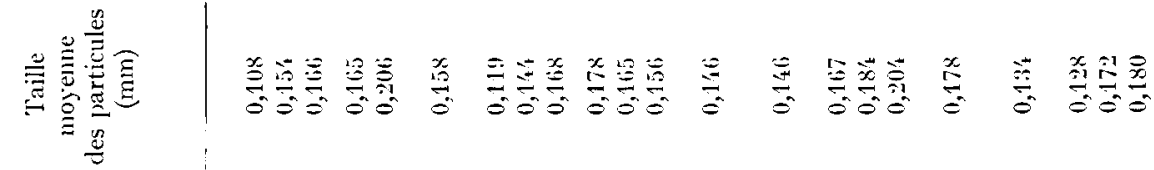

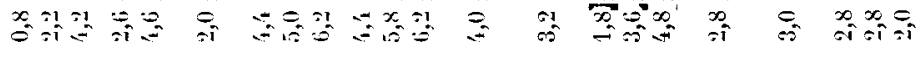

है

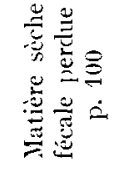

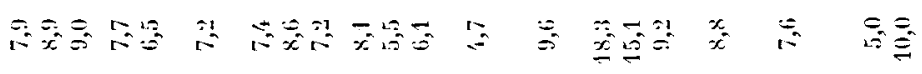

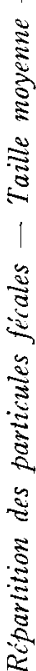

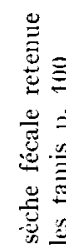

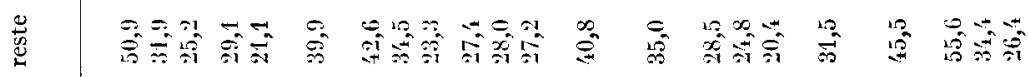
O

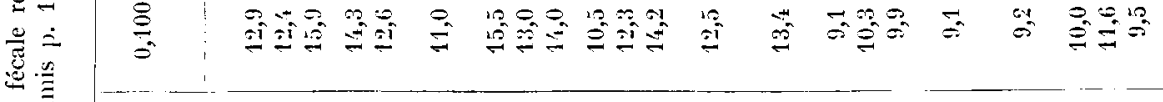

递

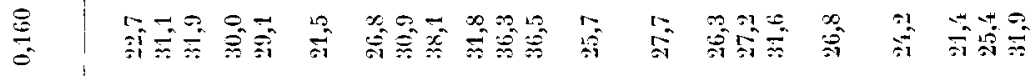

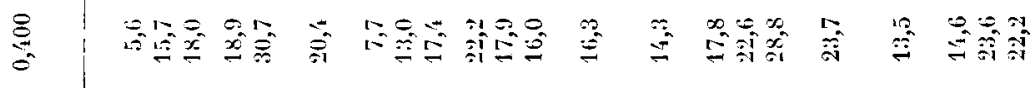

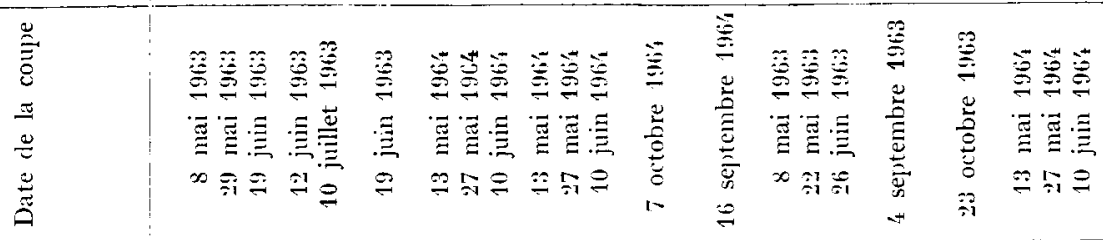

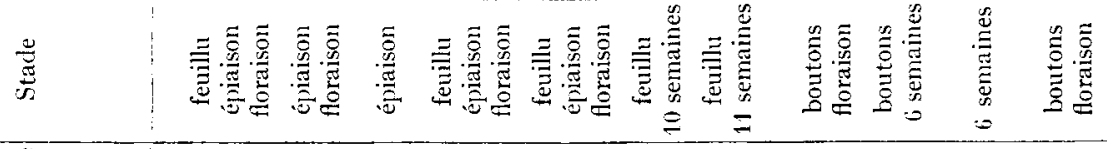

范 $\mid+-\infty+\infty-\infty+\infty-$

諾

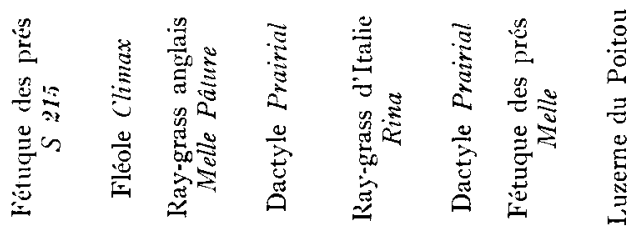

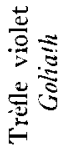


Le tableat 2 montre que la taille moyenne des particules est variable : de $0,108 \mathrm{~mm}$ pour la fétuque au stade feuillu à $0,206 \mathrm{~mm}$ pour la fléole à la floraison. Elle augmente au cours du I $^{\text {er }}$ cycle de croissance dans tous les échantillons de fèces, alors que la proportion de feuilles et que la digestibilité diminuent. Cet accroissement est le plus sensible pour les fèces de fétuque des prés.

Les coefficients de corrélation entre la taille des particules fécales et les caractéristiques du fourrage et des fèces sont donnés dans le tableau 3. Les liaisons sont plus étroites lorsqu'on élimine le ray-grass d'Italie: par exemple le coefficient de corrélation entre la taille moyenne des particules et la digestibilité de la matière organique est de $-0,624$ au lieu de $-0,582$. Le comportement aberrant du ray-grass d'Italie est peut-être dî̀ à la présence de repousses dans le pied.

La taille moyenne des particules fécales varie en sens inverse de la digestibilité de la matière organique (voir fig. I) et de la digestibilité de la cellulose brute des fourrages $(r=-0,582$ et $-0,569)$. En I 963 , elle est plus élevée, à digestibilité égale, pour la fléole que pour la fétuque. La rapidité de son évolution en fonction de la digestibilité au cours du I ${ }^{\text {er }}$ cycle varie selon les espèces. Elle est plus faible pour des fourrages d'automne que pour des fourrages de printemps de même digestibilité, sauf pour le dactyle.

La taille moyenne des particules fécales augmente avec la teneur en cellulose brute et en matière organique indigestible des fourrages; elle augmente également avec la teneur en cellulose brute des fèces $(r=+0,760)$, mais varie en sens inverse de leur teneur en matières azotées $(r=-0,593)$.

Elle varie en sens inverse de la quantité de matière sèche ingérée par jour et par kilogramme de poids métabolique $\left(\mathrm{P}^{0,75}\right)$, tout au moins pour les graminées (sauf pour le ray-grass d'Italie). Ia relation n'est pas aussi étroite pour les légumineuses; cependant le fait que, pratiquement, les moutons n'étaient pas alimentés ad libitum lorsque les deux premiers échantillons de fèces de luzerne ont été prélevés, pourrait en être l'explication.

\section{Nature des tissus végétaux non digérés}

Afin de compléter les observations faites sur les 22 échantillons déjà cités, un échantillon de fèces de fétuque élevée du $\mathrm{I}^{\text {er }}$ cycle a été examiné.

Nous avons étudié principalement les particules fécales de taille supérieure à $0,400 \mathrm{~mm}$, mais nous avons observé que les particules plus fines comprennent des spirales de vaisseaux plus ou moins déchiquetées et des débris difficilement identifiables (bactéries, débris végétaux et cellules du tube digestif) en grand nombre.

Dans tous les échantillons les particules de taille supérieure à $0,400 \mathrm{~mm}$ sont composées principalement de tissus lignifiés, en particulier de vaisseaux, d'épiderme et de parenchyme.

Mais des différences apparaissent entre les fèces de graminées et de légumineuses de printemps : dans ces dernières nous avons trouvé peu d'épiderme, seulement quelques lambeaux au début de la croissance du trèfle (ainsi que des fragments d'épiderme des graminées étrangères). Par contre, dans les fèces de toutes les graminées nous avons observé une proportion importante d'épiderme, proportion qui est sensiblement la même pour les différences espèces.

A l'automne tous les échantillons de fèces, de légumineuses comme de gra- 
minées, renferment des fragments d'épiderme, avec des vaisseaux et des cellules sous-épidermiques cellulosiques dans lesquelles nous avons vu, semble-t-il, des chloroplastes (dactyle, luzerne). Nous avons noté également la présence de quelques lambeaux d'épithélium digestif dans les fèces de luzerne.

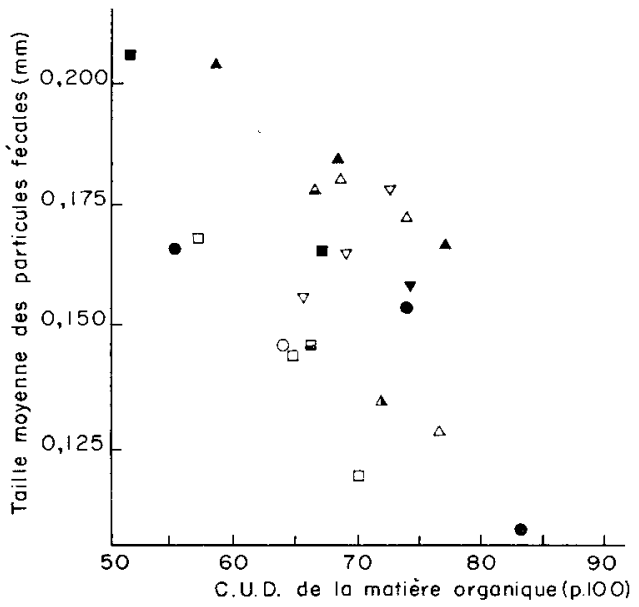

FIG. I. - Relation entre la laille moyenne des particules técales et le coefficient de digesibibitié de la malière organique.

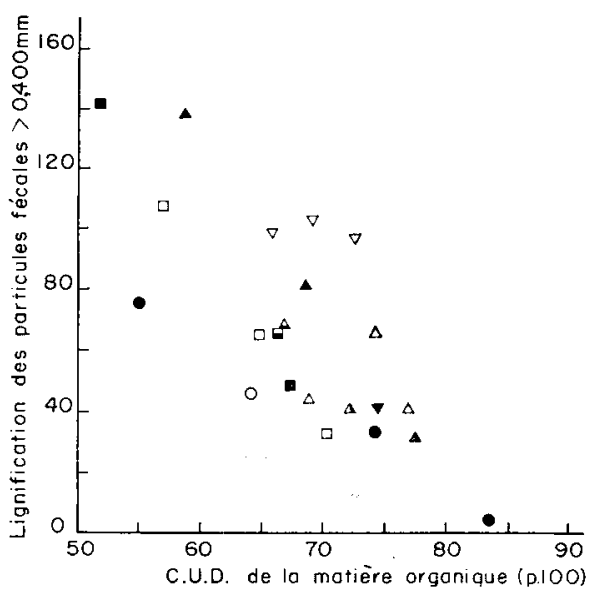

FIG. 2. - Relation entre la lignification des particules técales $>0,400 \mathrm{~mm}$ et le coefficient de digesibilité de la matière organique.

$\checkmark$ Ray-grass d'Italie

- Luzerne $\mathrm{I}^{\mathrm{er}}$ cycle

$\Delta$ Luzerne $3^{\text {e }}$ cycle

$\Delta$ Trèfle

A Luzerne $4^{e}$ cycle

\section{Lignification des particules fécales}

Nous avons évalué la proportion de tissus lignifiés dans les particules de taille supérieure à $0,400 \mathrm{~mm}$ par une note variant de o à Io. La différence entre les deux notes attribuées à chaque préparation est très faible; aussi, bien que la méthode ne soit pas très précise, elle permet de comparer les échantillons. La moyenne des 20 notes pour chaque échantillon, ainsi que leur variabilité, sont données dans le tableau 2.

Cette note de lignification est très variable, de 0,8 à 6,2 : elle augmente au cours du I er cycle sauf pour le trètle. Elle est plus élevée pour le dactyle et pour la luzerne que pour la fétuque, sous réserve d'une variation due à l'année.

En multipliant cette note par la quantité de particules de taille supérieure à $0,400 \mathrm{~mm}$, exprimée en p. Ioo de la matière sèche fécale, on obtient une estimation de la lignification des fèces. Les coefficients de corrélation entre la lignification des fèces et les caractéristiques du fourrage et des fèces sont donnés dans le tableau 3.

L, a lignification des fèces s'accroît tandis que la proportion de feuilles diminue et que le fourrage vieillit, sauf pour le trèfle. Elle varie en sens inverse de la digestibilité (sauf pour le trèfle) (fig. 2), et dans le même sens que la teneur en cellulose brute du fourrage et des fèces. 
Nous constatons une même évolution générale de la lignification avec l'âge pour toutes les espèces, mais il existe des différences entre elles. La lignification des fèces de luzerne est plus importante que celle des fèces de graminées de composition ou de digestibilité comparables, sauf pour la fléole à la floraison. La lignification des fèces de fétuque est plus faible que celle des fèces de fléole, en Ig63.

TABLEAU 3

Corrélation entre la taille et la lignification des particules fécales et les caractéristiques du fourrage et des fèces

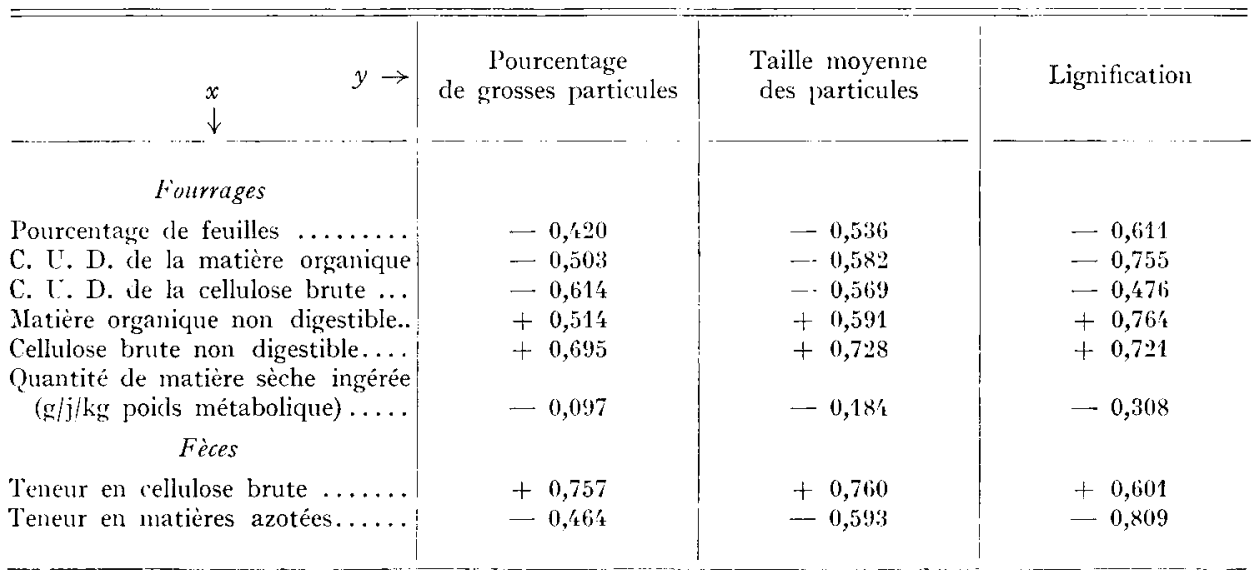

$r$ significatif à partir de 0,1 '́z $\mathrm{P}<0,05$

Les fèces de fourrages d'automne sont plus lignifiées que celles des fourrages de printemps, à proportion de feuilles égale ; à digestibilité égale, c'est l'inverse qui se produit, sauf pour le dactyle.

\section{DISCUSSION}

Malgré le caractère arbitraire ou l'imprécision des méthodes que nous avons employées, les variations mises en évidence sont suffisamment importantes pour comparer les résultats entre eux.

\section{Nature des particules fécales}

Nos résultats concernent essentiellement les fragments végétaux de taille supérieure à $0,400 \mathrm{~mm}$; nous n'avons pas étudié les particules plus fines qui comprennent aussi des débris d'origine endogène. Les grosses particules sont composées essentiellement de tissus lignifiés (vaisseaux, sclérenchyme), mais aussi de tissus cellulosiques (parenchyme) et d'épiderme en quantités variables. 
Dans tous les échantillons de fèces nous trouvons des tissus lignifiés, ce qui montre bien que les membranes lignifiées sont indigestibles en majeure partie ainsi que les analyses chimiques l'avaient montré. Au cours de la croissance, la proportion de tissus lignifiés dans la plante augmente, le fourrage devient moins digestible, et corrélativement les particules fécales deviennent de plus en plus lignifiées (sauf pour le trèfle).

Mais nous trouvons également de petites plages de tissus cellulosiques qui accompagnent ces tissus lignifiés; ceci confirme le fait que les membranes cellulosiques ne sont pas obligatoirement digérées. Lorsqu'une même particule est composée à la fois de tissu lignifié et de tissu cellulosique, il est possible que le premier ait protégé le second des attaques bactériennes. Cependant certaines particules comprennent uniquement des membranes cellulosiques. Nos résultats sont en accord avec ceux de REgal (I960) et Heliström (I965), tandis que Drapala, RAymond et Crampton (I947) et STEPPLER (I95I) ne retrouvent pas de tissus cellulosiques dans les fèces : cela dépend probablement du stade et de l'espèce étudiés.

Par ailleurs, des fragments d'épiderme sont présents en quantité variable dans les particules fécales. Au cours du I er cycle de nombretux fragments d'épiderme de graminées sont excrétés tandis que ceux de légumineuses sont rares. Cependant Hercus (I960) et Martin (I962) ont identifié des fragments d'épiderme dans les fèces de luzerne et de trèfle. Si nous n'en avons pas trouvé, ou seulement en faible quantité, est-ce parce qu'ils étaient trop fins, de taille inférieure à $0,400 \mathrm{~mm}$, ou bien parce que la cutine n'était pas déposée en couche épaisse aux stades étudiés? Nous avons constaté une différence avec les fèces récoltées à l'automne : la présence de fragments d'épiderme trouvés en grand nombre dans les fèces de légumineuses et de graminées, sur lesquels adhéraient encore des cellules sous-épidermiques avec parfois des chloroplastes, est un caractère important des fèces d'automne. Cela joint à la présence éventuelle de lambeaux d'épithélium digestif (luzerne $4^{\mathrm{e}}$ cycle) pourrait expliquer l'accroissement de la teneur en azote dans les fèces d'automne eu égard à la digestibilité de la plante (cf. par exemple MrNson et KEMP, I96I).

\section{Taille des particules fécales}

La taille moyenne des particules est relativement faible, de o, Io8 à 0,206 mm. Il est nécessaire que le fourrage soit réduit en petites particules pour passer du rumen dans le feuillet. Nous avons commencé l'examen de contenus de feuillet afin de vérifier cette hypothèse.

L'augmentation de la taille des particules fécales au cours du I er cycle avait déjà été observée par STTEPPler (I95I), DrapalA, RAYMOND et CRAMPTON (I947), et HeLLsTröm ( I965). Les feuilles donnent naissance à de petites particules, en particulier les feuilles de légumineuses, aussi la diminution de la proportion de feuilles contribue à l'augmentation de la taille des particules.

L'étude microscopique des fèces permet d'interpréter les variations de la composition chimique des fèces et de la digestibilité des fourrages. Nous la compléterons par l'observation des fines particules de taille inférieure à $0,400 \mathrm{~mm}$, par l'examen de contenus du tube digestif (du feuillet notamment), et par l'étude microscopique de la lignification des fourrages. 


\section{SUMMARY}

\section{PLANT PARTICLES IN THE FAECES OF SHEEP}

I. Twenty-two samples of faeces of sheep fed on 5 grasses and 2 legumes cut at different stages of growth and from the first, third and fourth cycles, were studied (table. I). The size of the particles in faeces was measured, and the nature and lignification of the plant particles in faeces were observed from microscopical examination.

2. The distribution of particles in faeces on 3 screens with mesh $0.400,0.160$ and $0.100 \mathrm{~mm}$, respectively, and at the bottom of the seive is shown in table 2 . It differed during growth of the forage. The percentage of dry matter of faeces retained by the 3 meshes increased regularly during the first cycle.

The average size of particles in faeces was small : it ranged from 0.108 to $0.206 \mathrm{~mm}$. In all samples of faeces it increased during the first cycle. It varied inversely with digestibility of organic matter (fig. I) and of crude fibre of the forages $(r=-0.582$ and -0.569 ) (table. 3).

3. In all samples the particles in faeces larger than $0.400 \mathrm{~mm}$ were composed essentially of lignified tissue (vessels, sclerenchyma). The presence of shreds of epidermis depended on the stage of growth and on the species studied. They were found in all samples of faeces from sheep given grasses but only in the third and fourth cycles with legumes.

4. The proportion of lignified tissue in the large particles increased as the proportion of leaf diminished and the plant grew older. It varied inversely with digestibility (fig. 2) and directly with crude fibre contents of forage and of faeces (table. 3 ).

5. The presence of epidermis with some sub-epidermal cells in faeces from herbage cut in autumn could explain the increase in nitrogen content of faeces in autumn, with due regard to the digestibility of the plant ingested.

\section{RÉFÉRENCES BIBLIOGRAPHIQUES}

Adams L., O'Regan W. G., Dunaway D. J., 1962. Analysis of forage consumption by fecal examination. J. Wildlife Mgmt., 26, 108-I11.

Croker B. H., 1959. A method of estimating the botanical composition of the diet of sheep. Nere Zeal. J. agric. Res., 2, $72-85$.

Drapala W. J., Raymond L. C., Crampton li. W., I947. Pasture studies XXVII. The effects of maturity of the plant and its lignification and subsequent digestibility by animals as indicated by methods of plant histology. Sci. Agric., 27, 36-41.

Hellström N., i955. A study in digestion. I. Analyses of feces from sheep. Kungl. Lantbr. Ann., 22, 9-16.

Hellström N., 1958. A study in digestion. II. Further analyses of feces from sheep. Kungl. Lantbr. Ann., 24, 49-54.

Hellström N., Aamisepp M., 1965. Digestion. III. Faecal analyses and digestibility. J. Sci. Food. Agric., 16, $27-33$.

Hercus B. H., 1960. Plant cuticle as an aid to determining the diet of grazing animals. Proc. 8th internation. Grassl. Congr. 1960, England, 443-447.

JARRIGE R., I965. La composition des fèces de mouton et sa relation avec la digestibilité du fourrage. Proc. 9th internation. Grassl. Congr. ig 65 , São-Paulo.

Johansen D. A., 1940. In Plant Microtechnique, 80-82. Mc Graw-Hill Book Company. New York.

Martin D. J., r962. Analysis of sheep diet utilizing plant epidermal fragments in faeces samples. In CRISP D. J., Grazing in terrestrial and marine environments. British Ecological Society Symposium, 4, I73-188. Blackwell, Oxford.

Minson D. J., KemP C. D., 196r. Studies in the digestibility of herbage. IX. Herbage and faecal nitrogen as indicators of herbage organic matter digestibility. J. brit. Grassl. Soc., 16, 76-79.

Regal V., I960. The evaluation of the quality of pasture grasses by the microscopic method. Proc. 8th internation. Grassl. Congr., ,1960 England, 522-524.

Steppler H. A., I95I. Pasture studies XXXI. Lignification studies with various grass species. Sci. Agric., 31, I-14.

Srork G. M., 196i. Microscopic analysis of faeces, a technique for ascertaining the diet of herbivorous mammals. Aust. J. biol. Sci., 14, I 57-164. 